\title{
Los objetos de adorno en «piedras verdes» de la Península Ibérica
}

\author{
ANA Fernández Vega \\ EnRIQue PÉrez Cañamares
}

La presencia de adornos realizados en «piedras verdes», en numerosos yacimientos de etapas eneolíticas o calcolíticas, fundamentalmente, aunque tampoco podemos excluir los de momentos inmediatamente anteriores, neolíticos, tanto en la Península Ibérica como en otras regiones europeas, ha llamado la atención de los prehistoriadores desde los comienzos de la historia de la investigación arqueológica.

Este ha sido también nuestro caso, ya que con motivo de trabajos anteriores ${ }^{1} y^{2}$, nos hemos encontrado con la presencia de objetos de adorno realizados en estas piedras verdes conocidas bajo el término genérico de «callais» o calaíta.

No queremos en esta ocasión hacer un análisis concreto de los yacimientos en los que aparecen estos objetos, sino más bien realizar un planteamiento más general, una especie de puesta al día de lo que hasta el momento se ha escrito sobre estas "piedras verdes", y esto, en 10 que se refiere a cuatro aspectos fundamentales:

1. Naturaleza y composición.

2. Origen y procedencia.

3. Significación.

4. Contexto arqueológico y Cronologia.

A. Fernandez Vega y K. Galan Saulnier: 7-26, 1986.

2 A. Fernandez Vega y E. Pérez Cańamares: e. p. 


\section{NATURALEZA Y COMPOSICIÓN}

Los primeros planteamientos sobre la naturaleza de estas piedras verdes aparecen con Damour en 1864, momento en el que se plantea, por primera vez "sobre el papel» algo más que las meras referencias a su existencia.

En esta fecha, Damour ${ }^{3}$ realiza los primeros análisis acerca de las diferencias y afinidades entre las turquesas orientales o turquesas propiamente dichas, y el "callais", término que -como veremos más adelante - engloba a su vez una buena variedad de materiales distintos, pero que en ese momento, e incluso hasta bastante después, se aplicó a todas aquellas piedras verdes que no eran turquesas orientales, e incluso en ocasiones a éstas también.

Las definiciones de Damour establecen no sólo las diferencias y afinidades de las dos materias, sino también la composición y características de ambas:

«...La turquoise, en effet, es plutot opáque que traslucide, sa couleur habituelle est le bleu celéste plus ou moins foncé; sa dureté, sa densité sont superieures à celles du noveau mineral. J'ajouterai que la turquoise orientale doit sa couleur à l'oxyde de cuivre, tandis, que la teinte verde de la nouvelle matérie me parait due l'oxyde de fer".

"Tandis que la turquoise orientále contient deux equivalents d'alumine pour un à d'àcide phosphorique et cinq d'eau, l'autre minéral ne contendrait qu'un seul élement d'eus."

Ahora bien, de esta definición se deduce claramente que la otra materia de la que habla Damour son las variscitas férricas, una de las muchas variantes incluidas bajo el término genérico de "callais". Como observa en las fórmulas químicas de estas materias: $\mathrm{Cu}(\mathrm{Al} \mathrm{Fe})\left(\mathrm{PO}_{4}\right)_{4}$ $(\mathrm{OH})_{8} 4 \mathrm{H}_{2} \mathrm{O}$ y $(\mathrm{Al} \mathrm{Fe}) \mathrm{PO}_{4}-2 \mathrm{H}_{2} \mathrm{O}$, respectivamente, en ambos casos se trata de minerales compuestos básicamente de fosfatos de aluminio pero en las variscitas los óxidos de cobre han sido sustituidos por óxidos de hierro, y la proporción del ácido fostórico en relación al óxido de aluminio es el doble que en las turquesas.

${ }^{3}$ M. Damour: 936-940, 1864. 
En esta línea de "clarificación", Cazalis de Foundouce ${ }^{4}$, en 1880 , delimita las diferencias entre turquesas, variscitas y lo que él denomina «nouvelle substance", refiriéndose a la calaíta o "callais".

A pesar de ello, las denominaciones posteriores siguen usando este término genérico para designar elementos de muy diferente composición, ya sea porque no se realizan análisis químicos de los materiales, ya sea por otras razones, una de las cuales podría ser un error existente en la definición de Damour, por lo que se refiere a la tonalidad de ambas sustancias. El habla de color verde para el "callais" y azul para la turquesa, cuando en realidad estas diferencias son inexistentes. En la definición de Church ${ }^{5}$ se afirma claramente:

"La turquesa oriental está presente en zonas delimitadas de Irán, India y Turquia. Su color oscila desde el azul cielo, al azul claro, al verde azulado, al verde, y cuando se tornan verde mate se las denomina turquesas muertas. Sus propiedades físicas son muy características y poco comunes. Tiene una dureza de grado 6 , de grano fino, aunque también se presente en pequeños cristales, y es de fractura concoide, débil brillo céreo, y bastante frágil. Se encuentra en masas formando filones, no se funde con soplete y en los ácidos se disuelve sin efervescencia y sin dejar residuo".

$\mathrm{Y}$, por su parte, Level ${ }^{6}$ en su texto sobre les "pierres precieuses", dice que el color de las variscitas puede ser: «vert-clair, bleu-vert, vertjaune intense, jaunâtre ou blanchâtre". Como veremos, poco diferente de las gamas con que aparece la turquesa auténtica.

Para mayor confusión, el problema no se reduce a las turquesas y variscitas, ya que bajo este término de "callais", conocido desde Plinio, se han venido agrupando - como ya hemos apuntado- otra gran variedad de compuestos con distintas características físicas y químicas, tales como malaquitas, talcos, micas, amblygonitas, wavelitas, lepidolitas, etc. Todas estas materias tienen una característica común desde el punto de vista físico: el color verde, que es la que ha dado lugar a toda la confusión, y otra desde el punto de vista cultural: son objetos de adorno.

La confusión entre variscitas, turquesas y toda otra serie de minerales de apariencia similar, se incrementa si tenemos en cuenta que la práctica de la falsificación de turquesas es muy común desde la antigüe-

\footnotetext{
4 M. Cazalis de Foundouce: 314-329, 1880.

5 A. H. CHURCH y otros: 1924.

6 T. et D. LEVEL: 479, 1980.
} 
dad. Ahora bien, los análisis evitarán en lo sucesivo los errores, tanto en este sentido, como en el de los diferentes materiales con propiedades físicas aparentemente comunes, debido a la componente básica de hidratos de aluminio fosfatados.

Como resultado de la mayor frecuencia de análisis de estos objetos, se puede ver que la mayoría de los materiales pertenece al grupo de las variscitas, mientras que son mucho menos frecuentes las cloritas, malaquitas o talcos, que podrian considerarse «imitaciones» de ésta. También hay turquesas orientales, aunque escasas.

De todo esto se desprende que el término "callais" o calaíta podría utilizarse como sinónimo un tanto arbitrario, de las variscitas de distinta composición, pero sin que ello excluya la existencia -aunque minoritaria- de otro tipo de piedras.

\section{ORIGEN Y PROCEDENCIA}

En este punto, en general, los autores han tendido a establecer una relación directa entre las actividades de prospección y extracción del cobre y el estaño y estas piedras verdes. Sin embargo, solamente las turquesas y las malaquitas están asociadas al cobre, y ya hemos visto que son minoritarias.

El primero en señalar una posible relación directa entre los adornos de piedra verde y la explotación del estaño, como consecuencia de la metalurgia del bronce, fue Capitan ${ }^{7}$, que excavó en dos yacimientos franceses: Echasiéres (Allier) y Montebras (Soumans). En el primero, junto a pozos de extracción de pegmatitas estanníferas -que podrian presumirse prehistóricas- existía un mineral secundario, un flúo-fosfato de aluminio de color verdoso: la amblygonita que, en ocasiones, tomaba un color azul similar al de las turquesas. Explotaciones similares existian en Montebras, en donde se asociaba a las citadas pegmatitas, la montebrasita que es una variedad de la amblygonita, de tonos azulados y en cuya composición química parecen existir óxidos de cobre, elemento éste que lo asemeja en gran medida a las turquesas orientales. Cazalis de

M. Capitan: 220-222, 1926. 
Foundouce ${ }^{8}$ habla de la existencia de afloramientos de peganitas, variscitas y fischeritas, materiales que habían sido incluidos bajo la denominación de "callais" en las zonas europeas de Sajonia, Silesia y Montes Urales.

Variscitas aparecen también en Austria, Checoslovaquia y Alemania, así como en un pequeño afloramiento en Pannecé-Nantes y en las zonas de Tras-os-Montes y Beira Alta en Portugal, como señalan A. Huet y B. Gonçalbes ${ }^{9}$ que también citan yacimientos de este mineral en Almeria (La Encantada, Adra) y Palazuelo de las Cuevas (Zamora). Por su parte, M. ${ }^{2}$ Josefa Villalba ${ }^{10}$ dice que se han identificado en las galerias de Can Tintoré en Gavá (Gerona) hasta diez minerales del tipo fosfatos, entre los que destaca la variscita en tres de sus variedades, la turquesa, la strengita, insertas en la base de pizarras alumínicas que forman el yacimiento, cuyas galerias mineras conocidas alcanzan cerca de 50 metros de longitud y 18 de profundidad.

Arribas y otros ${ }^{11}$ destacan la importancia del yacimiento zamorano: «...Un gran yacimiento de variscita se extiende por el oeste de la provincia de Zamora a lo largo de casi $30 \mathrm{~km}$ ", señalando además el hecho de su explotación en etapas prehistóricas: «...La explotación del yacimiento en época antigua no ofrece duda ya que al SW de Palazuelos de las Cuevas... se reconocen varias excavaciones con abundantes vetillas y con secciones de variscita".

Para López Plaza, la relación de estas piedras y la explotación del cobre es evidente: «EI SO de la provincia de Zamora constituiría un centro comercial de indudable importancia en conexión con los primeros prospectores y explotadores de los metales. En este sentido, hemos de destacar que cercano al yacimiento de variscita se encuentra el de cobre de Muga de Alba" ${ }^{12}$.

La existencia de la materia prima de estos adornos en "piedras verdes" en la Peninsula lbérica nos conduce a otro de los problemas sobre los que se ha polemizado bastante: el origen local o extrapeninsular de tales piedras.

- Vid. nota 4: 321.

9 A. Huet y B. Goncalves: 4-22, 1980.

${ }^{10}$ M. J. VillLalba: 1986.

1 A. ARRIBAS y otros: 115-132, 1971.

i2 S. LOPEZ Plaza: 4, 1982. 
López Plaza ${ }^{13}$ confirma «el origen local y autóctono de determinadas materias primas muy utilizadas durante el Calcolítico para fabricar objetos de adorno", cuando habla de la variscita, y añade aún más: "...en algunos casos, las sustancias empleadas... pueden tener su origen en las inmediaciones de los lugares donde se encuentran".

Los descubrimientos de Can Tintoré parecen confirmar estas aseveraciones, y aún más, podrian explicar incluso las turquesas que pudieran identificarse en regiones peninsulares.

En este sentido, Vázquez Varela dice: “El origen local de los minerales hace innecesario suponer relaciones comerciales con áreas lejanas extrapeninsulares" ${ }^{14}$.

Ana M. ${ }^{a}$ Muñoz no considera la posibilidad de un comercio extrapeninsular: «...no creo en un comercio a larga distancia, sino más bien en posibles recursos locales» ${ }^{15}$, y tampoco Huet y Gonçalves son partidarios de un origen comercial con Oriente: «A existencia na Península lbérica das espécies mineralógicas utilizadas na confeçáo dos elementos de adorno de cor verde leva-nos a rejeitar a hipotese de relaçoes comerciais con Oriente» ${ }^{16}$.

La posibilidad de una doble vía queda abierta años después para Guitián Rivera y Vázquez Varela ${ }^{17}$, quienes de alguna manera sintetizan ambas opiniones: "Hoy a la luz de los análisis físicos y químicos ha de precisarse la sustancia empleada en las cuentas y ha de buscarse con posterioridad una procedencia independiente para cada uno de los minerales usados en la realización de las mismas".

Como vemos a lo largo de este recorrido, parece que la tesis occidentalista sobre el origen de estas piedras es la más aceptada, aun cuando no sea excluida la posibilidad de una doble via o procedencia.

La evidente diferencia entre la abundante difusión de objetos de estas materias y los escasos yacimientos de materias primas conocidas hasta el momento, ha dado lugar también a diferentes interpretaciones. $Y$ así, mientras que López Plaza considera ${ }^{18}$ que es factible hablar de un comercio de metal asociado al comercio de piedras verdes en un

\footnotetext{
13 Vid. nota 12: 4.

14 J. M. VazQuez VAREla: 28, 1975.

15 A. M. MuÑoz AMiLIBIA: 354, 1971.

16 Vid. nota 9: 15.

"F. Guitian Rivera y J. M. Vazouez Varela: 187, 1975.

${ }_{18}$ Vid. nota 12: 4.,
} 
marco interpeninsular, Ana $M .{ }^{a}$ Muñoz ${ }^{19}$ cree que los afloramientos de variscitas y otros fosfatos alumínicos pueden haber sido muy extensos en cantidad pero poco potentes, de forma que una vez explotados y agotados, hoy no tendriamos posibilidad, o al menos nos resultaría muy difícil, su identificación.

Sin embargo, no parece muy aceptable la hipótesis de una gran profusión de pequeños afloramientos repartidos por el territorio peninsular que darian lugar a una minería local que explicaria la extensión de la presencia de estos adornos en piedra verde en la Península Ibérica. El señor Mata, del Instituto Geográfico y Minero, nos ha confirmado que no es probable la existencia de tales afloramientos, pero que la proporción de yacimientos de piedras verdes en la Península lbérica aumentaría considerablemente si se incluyen talcos, micas, etc., de características formalmente similares (color) a las variscitas.

El hecho de que el mineral utilizado sea de procedencia peninsular -y los hallazgos de Gavá parecen confirmarlo- no implica que el material no evidencie unas relaciones mediterráneas muy acusadas que pueden no ser comerciales en el sentido de importación de material, pero sí culturales.

Dentro del contexto eneolítico peninsular, sí parece evidente la existencia de una corriente comercial local, en un sentido restringido del término. Tal vez en las zonas en que dicha corriente comercial o de intercambio no existe o es muy débi, es decir, en zonas con asentamientos marginales de poblaciones eneolíticas, pudo recurrirse a materiales diversos, distintos de las variscitas que son las más extendidas y utilizadas, todas ellas en color verde. Esto nos lleva a pensar que es precisamente esta característica: el color, y no sus características mineralógicas, ni tampoco su posible asociación con minerales de cobre, lo que les lleva a buscar estas piedras para la fabricación de objetos de adorno, usados además generalmente en contextos funerarios. Y asi, nos metemos directamente en el apartado dedicado al posible significado cultural de este color.

19 Vid. nota 15: 354 . 


\section{SIGNIFICACIÓN}

En su obra, Tardy y Diva Level ${ }^{20}$ dicen, refiriéndose a la turquesa: "...était connue dans l'Antiquité, certainement 3.000 ans avant notre ére. Les Pharaons en faisaient orner leux bijoux et en faisaient des talismans".

Aun cuando en ésta y en otras referencias, los investigadores se retieren a las turquesas, to hacen siempre en relación con las tonalidades, y por ello, nos sirve para nuestra interpretación de otras piedras que no son en realidad más que imitaciones de ésta.

Church habla de un uso mágico y religioso de la turquesa, pero en otro contexto bien diferente, cuando dice:

"Turquoise, also met with apreciation in the New World. The aztecs were using it at the time of the Spanish Conquest in the form of mosais applied to wooden maks of their gods, combined with shell inlays for eyes ant teths". ${ }^{21}$

También Serpa Pinto ${ }^{22}$, en su trabajo sobre cuentas empleadas como amuletos, cita una de color verdoso como posible "relicario".

Por su parte, Pérez de Barradas ${ }^{23}$ alude al simbolismo del verde en el antiguo Egipto y también afirma que este color simbolizó la primavera y la tierra en época clásica. También, según este autor, para los aztecas "el verde era representativo de Chal chiu Hiane, diosa del agua».

La turquesa fue considerada, según Church ${ }^{24}$, por persas y egipcios como una piedra del cielo y por tanto intensamente explotada. Dicha explotación iba destinada a la creación de amuletos funerarios para la otra vida:

"The majority of egiptian jewellery and almost all of their masterpieces were funerary objects, buried with the dead as an amuletc aid in the world to come".

${ }^{20}$ Vid. nota 6: 477 .

21 Vid. nota 5: 69.

22 R. DE SERPA PINTO: 1932.

23 J. Perez de Barradas: 24-43, 1967.

24 Vid. nota 5: 187. 
También la esmeralda, otra piedra de color verde tuvo un destino similar, para contextos funerarios:

"The ancient egyptian also regarded emerald, with is deep green colour unlike the green of anything else known to them as being mystical and magical... emerald were used in funerary jewellery for dead nobles" ${ }^{25}$.

Y aún hay más: cuando la esmeralda escasea, se la sustituye por imitaciones, pero no en cuanto a su composición quimica se refiere, sino en cuanto a su color que es precisamente lo que la relaciona con su función funeraria.

"The problem however was that emerald were in such shot supply in Egypt to compensate for their scarcity, green faience came to be used skilfully simulating the majesty of emerald colour" ${ }^{26}$.

No sólo el color, sino también la forma, parece haber tenido una significación especial en el destino de las piedras de adorno. W. Deonna, en su trabajo sobre "Le simbolisme de l'oeil» menciona la siguiente función para las cuentas de vidrio de forma oval: "Les perles ou grains d'enfilage qui composent les colliers ont souvent une utilité prophylactique» ${ }^{27}$. $Y$ en relación a las cuentas de pasta vitrea en uso durante el II Milenio a.C. en Egipto, afirma que no sólo por su forma o por el sistema de uso, sino que por su color azul, tienen un carácter profiláctico: "Ces perles sont souvent bleues, couleur prophylactique" ${ }^{28}$.

Hay numerosas alusiones a las funciones de las turquesas en las culturas mediterráneas en general y en Egipto en particular. No queremos sino señalar que de la misma forma que se extendió un determinado ritual que protegía a los muertos en cuevas o megalitos, que colocaba junto a ellos objetos de uso cotidiano, aparentemente al menos para servir al difunto en la otra vida, podemos suponer que otro elemento del ritual funerario, las piedras de color verde, protectoras de la salud, profilácticas y mágicas pueden ser el testimonio de un proceso de aculturación que llega a la Península Ibérica con la metalurgia.

\footnotetext{
25 Vid. nota 5: 53.

26 Vid. nota 5: 53.

27 W. DEONNA: $195,1965$.

28 Vid. nota 27: 196.
} 


\section{CONTEXTO ARQUEOLÓGICO Y CRONOLOGIA}

No es nuestro objetivo hacer aqui un análisis exhaustivo de los materiales con los que aparecen asociados estos adornos realizados en "piedras verdes", pero sí el de hacer una breve referencia tanto a los tipos de adornos más frecuentes en esta materia como a los demás objetos que los acompañan, comenzando precisamente por aquéllos de idéntica finalidad a las cuentas que son el tema de este trabajo, es decir, los adornos. En general, lo más frecuente y casi lo único dentro de este grupo, son las cuentas de collar, aun cuando existen también algunos colgantes.

Las cuentas ofrecen diversas variantes en cuanto a formas, tales como esferoidales, discoidales, y sobre todo las denominadas "de tonelete", "bitroncocónicas" o "de oliva». En cuanto a los materiales en que están elaboradas, podemos destacar los diferentes tipos de piedra pulimentada, así como el hueso y la concha, ya sean éstas simplemente perforadas o bien recortadas, y también materias locales, tales como la madera y el azabache.

Por lo que se refiere al resto de los materiales con que aparecen estos adornos, podemos citar numerosas puntas de flecha en piedra tallada, con casi todas las variedades tipológicas que éstas presentan, los cuchillos y lascas, así como algunas raederas, raspadores, y en ocasiones, denticulados. Lo más frecuente, en cuanto a útiles de piedra pulimentada, son las hachas, y en hueso hay punzones, espátulas y agujas, así como botones con perforación en " $\mathrm{V}$ ", aunque menos abundantes. Esporádicos son algunos idolos de hueso, y el metal es generalmente escaso, presentándose en forma de algunos puñales de lengüeta y de remaches y un número algo mayor de punzones.

Las cerámicas ofrecen formas de cuencos, generalmente semiesféricos, algunas formas de carena baja y vasos de paredes rectas. Predominan las lisas y sólo en ocasiones llevan decoración a base de cordones. Hay algunas asas y mamelones.

Como se puede observar en este brevísimo repaso por los materiales más comúnmente asociados a la "calaíta", si bien puede hablarse en términos generales de una atribución a momentos calcolíticos - con todas las reservas que puedan plantearse sobre esta denominación, debido a las discusiones terminológicas sobre la cuestión, en las que en modo alguno pretendemos entrar aqui y ahora - no nos sería posible establecer una periodización, ni siquiera una clasificación cronológica más con- 
creta y delimitada. Esto implicaría, además de un estudio detallado del contexto material de cada uno de los yacimientos, así como de sus caracteristicas morfológicas, temas ambos que darian lugar a un trabajo mucho más amplio de lo que las limitaciones especiales nos imponen, un planteamiento que ya hemos expuesto en otras ocasiones ${ }^{29}$ sobre la cronología, o mejor sobre los indicios cronológicos que nos permiten establecer una hipótesis acerca de la periodización interna de este período.

Por todo ello, nos limitaremos aquí a resaltar el hecho de que también entre los materiales que acompañan a los objetos en "piedra verde", podemos ver diferencias evidentes en lo que a su atribución cronológica se refiere. De nuevo, nos encontramos con objetos que ofrecen «indicios» bastante antiguos dentro de esta etapa, pero también bastante recientes, como es el caso de las cerámicas con cordones o el del puñal de remaches.

Como siempre nos ocurre, es al llegar al final del trabajo cuando se nos plantean otra serie de preguntas y también de objetivos que ya no podemos cumplir, tal como sería el análisis de los yacimientos en los que aparecen los materiales citados como más atipicos dentro del conjunto, del que ya hemos hablado en líneas anteriores, y que nos llevaría de nuevo a un problema de fondo en el que nos movemos hace tiempo, como es el de la periodizacion interna de esta etapa.

Ponemos, pues, un punto y seguido a este tema, nunca un punto final, $y$, también como siempre, nos planteamos todo lo que necesitamos saber y lo poco que aún sabemos.

29 Vid. nota 1. 


\section{BIBLIOGRAFÍA}

Arribas, A. y otros, 1971: Estudio mineralógico de la variscita de Palazuelo de las Cuevas, Zamora (España). Salamanca. Studia Geológica II: 115-132.

CAPITAN, M., 1926: Les gisements d'etain en France. La question de la callais. Revue Anthropologique: 220-222.

Cazalis de Fondouce, M., 1880: De l'emploi de la callais dans l'Europe Occidentale aux temps préhistoriques. Lisbonne, C. R. Cong. Intern. Antrhop. Arch. Préh. 9.: 314-329.

ChANTREt, F. y otros, 1970: Analyses de quelques perles méridionales en «callais". B.S.P.F. 67: 216-219.

Chantret, F. y otros, 1970: Les perles en callais. Analyses de specimens du Midi de la France. Pyrenée 6: 29-37.

ChurCH, A. H. y otros, 1924: Precious Stones. Londres.

Damour, M., 1864: Sur la callais, nouveau phosphate d'aluminium hydraté recueilli dans un tombeau celtique du Morbihan. Compte-rendus des séances de l'Academie des Sciences LIX: 936-940.

Delibes de Castro, G. y Santoja, M., 1986: El fenómeno megalítico en Salamanca. Salamanca. Pub. Diputación Provincial.

Deonna, W., 1965: Le symbolisme de l'oeil. Paris. Edit. E. de Boccard: 192-196.

Fernández Vega, A. y Galán Saulnier, C., 1986: Las denominadas cuevas sepulcrales colectivas eneolíticas del País Valenciano y la Meseta. Boletín del MAN IV: 7-26.

Fernández Vega, A. y Pérez Cañamares, E. (e. p.): Cuevas de enterramiento en el Pirineo Catalán. 
Forestier, F. H. y otros, 1973: A propos de la "Callais". Découverte d'un gisement de variscite à Pannecé. Analyse de quelques uperles vertes" néolithiques. BSPF 70: 173-180.

Frazer, J. G., 1967: The Magic Art and the Evolution of kings. Vol. I de The Golden Bough.

Guitian Rivera, F, y Vazquez Varela, J. M., 1975: Análisis radiográfico de cuentas de calaita gallegas. Lugo. Boletin de la Comisión de Monumentos 9: 187-188.

HUEt, A. y Goncalves, B., 1980: Elementos de adorno de cor verde provenientes de estaçoes arqueológicas portuguesas. Importancia do seu studo mineralogico. Trabalhos do Instituto de Antropología Dr. Mendes Correa n..$^{\circ}$ 40: 4-22.

LEVEL, T. et D., 1980: Les pierres precieuses. Paris.

LOPEZ PLAZA, S., 1982: Aspectos arquitectónicos de los sepulcros megalíticos de las provincias de Salamanca y Zamora. Salamanca. Edic. Universal.

Mata, J. M., Plana, F. y Travería, A., 1983: Estudio mineralógico del yacimiento de fosfatos de Gavá. Madrid. Bol. Soc. Esp. Mineralogia: 257-258.

MiRo, J. I., 1870: Estudio de las piedras preciosas. Madrid.

MuÑoz AMILIBIA, A. M., 1965: La cultura neolítica catalana de los sepulcros de fosa. Barcelona. Inst. Arq. y Prehistoria Universidad. Publicaciones Eventuales $n^{\circ} 9$.

- 1971: La calaíta en el Pais Vasco. Munibe XXIII: 347-354.

Pérez de Barradas, J.: El color en la vida y en el arte de los pueblos. AMSEAEP XI Y XII.

Plaza y MATA, J., 1984: Los materiales del yacimiento neolítico de Can Tintoré, Gavá. Barcelona.

Salvado Canelhas, M. G., 1973: “Estudio radiográfico de "calaítas" portuguesas". Revista Guimares 83: 125-145.

SCHUMAN, W., 1983: Guia de las piedras preciosas y ornamentales. Barcelona.

Serpa PINTO, R. DE, 1932: Etnografía Arqueológica. 1. Antigas contas empregadas como amuletos. Porto. Instituto Antropología.

VÁzquez VARelA, J. M., 1975: Cuentas de "calaíta" en la Península lbérica: datos para la revisión del problema. Gallaecia. 1:27-30.

VeIgA FERREIRA, O. DA, 1951: Os artefactos prehistoricos de calaite e sua distribuçao em Portugal. Lisboa. Arqueologia e Historia V. 85-93.

VILASECA, S., 1971-72: A propósito de la calaíta o variscita. Ampurias 3334: $287-288$. 
Villalba, M. J. y otros, 1976: Can Tintoré, Gavá. En «El Neolitic a Catalunya". Pub. Dip. Barcelona.

VILlalba, M. J., 1986: Las minas neolíticas de Can Tintoré. Gavá. Barcelona. Pub. Generalitat de Catalunya. 